\title{
Effet de la salinité sur la floraison, la fructification et la qualité nutritionnelle des fruits du cultivar local Akikon de tomate (Lycopersicon esculentum Mill.) du Bénin
}

\author{
Eliane KINSOU ${ }^{1}$, Abdou Madjid AMOUSSA ${ }^{2}$, Armel Clément Goudjo MENSAH ${ }^{3}$, \\ Julien Koffi KPINKOUN ${ }^{1}$, Françoise ASSOGBA KOMLAN ${ }^{3}$, Hyacinte AHISSOU ${ }^{4}$, \\ Latifou LAGNIKA ${ }^{2}$ et Christophe Bernard GANDONOU ${ }^{*}$
}

\begin{abstract}
${ }^{1}$ Unité de Recherche sur l'Adaptation des Plantes aux Stress Abiotiques, les Métabolites Secondaires et l'Amélioration des Productions Végétales, Laboratoire de Physiologie Végétale et d'Etude des Stress Environnementaux, Faculté des Sciences et Techniques (FAST/UAC), 01BP526, Tri Postal, Cotonou, République du Bénin.

${ }^{2}$ Laboratoire de Biochimie et Substances Naturelles Bioactives, Unité de Biochimie et Biologie Moléculaire, Faculté des Sciences et Techniques, UAC, 04 BP 320 Cotonou, République du Bénin.

${ }^{3}$ Centre de Recherches Agricoles Plantes Pérennes (CRA-PP), Pobè, Institut National des Recherches Agricoles du Bénin (INRAB), Abomey-Calavi, Bénin.

${ }^{4}$ Laboratoire d'Enzymologie et de Biochimie des Protéines (LEBP), Institut des Sciences Biomédicales Appliquées (ISBA), Cadjèhoun, Cotonou, République du Bénin.

*Auteur correspondant ; E-mail: ganchrist@hotmail.com; Tel. (+229) 97396978
\end{abstract}

Received: 19-11-2020 Accepted: 30-03-2021 $\quad$ Published: 30-04-2021

\section{RESUME}

La tomate est l'un des légumes les plus consommés au monde et au Bénin. Il est largement établi que la salinité agit négativement sur la germination et la croissance et modifie considérablement la concentration des composés bioactives des légumes. Au Bénin, la tomate est cultivée partiellement sur les terres cultivables des régions côtières où la salinité du sol et de l'eau d'irrigation est une réalité. Cette étude visait à évaluer l'effet de la salinité sur la floraison, la fructification et la composition nutritionnelle des fruits de tomate du cultivar local Akikon. Les plantes de trois semaines ont été arrosées jusqu'à la maturité des fruits avec diverses concentrations de $\mathrm{NaCl}$ de $0,30,60$ et $120 \mathrm{mM}$. La date d'apparition des premières fleurs, la date de maturation des fruits ainsi que les teneurs en protéines des fruits n'ont pas été significativement affectées par la salinité alors que le nombre de fruits par plante, le poids des fruits, les teneurs en sucres solubles totaux et en sucres réducteurs subissent une réduction significative sous l'effet du $\mathrm{NaCl}$. Par contre, les teneurs en lycopène, en vitamines $\mathrm{B} 6, \mathrm{~B} 12$ et $\mathrm{C}$ ont connu une augmentation significative avec l'augmentation de la concentration de $\mathrm{NaCl}$. La salinité a donc réduit la production de tomate et amélioré la qualité nutritionnelle des fruits en augmentant notamment ses teneurs en vitamines et en lycopène.

(C) 2021 International Formulae Group. All rights reserved.

Mots clés: Salinité, teneur en nutriments, sucres, lycopène, vitamines. 


\title{
Effect of salt stress on flowering, fructification and fruit nutritional quality of Benin local tomato (Lycopersicon esculentum Mill.) cultivar Akikon
}

\begin{abstract}
Tomato (Lycopersicon esculentum) is one of the most important consumable vegetable in the world and in Benin. It is widely established that salinity acts negatively on germination and growth and considerably modifies the concentration of bioactive compounds in vegetables. In Benin, tomato is partially cultivated on arable lands in coastal regions where soil and irrigation water salinity is a reality. This study investigated the impact of salinity on flowering, fructification, and fruit nutrient contents of a local cultivar of tomato Akikon. Three weeks old plants were irrigated with four $\mathrm{NaCl}$ concentrations: $0 ; 30 ; 60$ and $120 \mathrm{mM} \mathrm{NaCl}$ till fruit ripening. The date of first flower appearance, the date of fruit ripening and fruit protein content were not affected by salinity; whereas fruit number, fruit fresh mass, fruit soluble sugars and reducing sugars contents were significantly decreased by increasing salinity. Moreover, lycopen and vitamins B6, B12 and C contents increased significantly when salinity concentration increased. Thus, salinity reduced tomato productivity but improved fruit nutritional quality by mainly increasing vitamins and lycopen contents.
\end{abstract}

(C) 2021 International Formulae Group. All rights reserved.

Keywords: Salinity, nutrients content, lycopen, sugars, vitamins.

\section{INTRODUCTION}

La tomate (Lycopersicon esculentum Mill.) est le légume le plus consommé dans le monde après la pomme de terre (Douakha et Guernine, 2013). Elle est cultivée sous presque toutes les latitudes et est consommée fraiche, préparée ou transformée en jus, pate ou sous forme de différentes sortes de sauces. Sa culture occupe près du tiers des surfaces mondiales consacrées aux légumes (Messai et al., 2006). La tomate est un aliment diététique riche en eau et pauvre en calories. Le fruit renferme aussi beaucoup d'éléments minéraux et de vitamines, dont la vitamine $\mathrm{C}$ (ou acide ascorbique). Le fruit mûr contient aussi des pigments de la famille des caroténoïdes dont le $\beta$-carotène qui possède une activité de provitamine $\mathrm{A}$ et le lycopène qui joue un rôle d'antioxydant dans l'alimentation humaine (Dembinska-Kiec et al., 2008) et dans la prévention de certains cancers (Bhowmik et al., 2012). La consommation de tomate sous de nombreuses formes (frais, soupes, jus, concentré, etc.) augmente constamment depuis quelques années, associée avec la découverte des propriétés anti-oxydantes du lycopène et de ses dérivés. L'intérêt nutritionnel de la tomate sur la santé est également lié à la présence de provitamines A (précurseurs de la vitamine A) et d'une large diversité de vitamines du groupe B. La tomate contient également de la vitamine C et représente un apport considérable de vitamine $\mathrm{C}$ pour notre organisme (Bhowmik et al., 2012). La coloration rouge de la tomate est due à l'accumulation de caroténoïdes principalement le lycopène et le bêta-carotène (McKinney, 2012). Ces caroténoïdes, fortement étudiés pour leur propriétés antioxydantes (Bhowmik et al., 2012), semblent impliqués dans la protection contre certaines maladies dégénératives (cancer de la prostate, maladies cardio- vasculaires).

$\mathrm{Au}$ Bénin, la tomate est cultivée partiellement sur les terres cultivables des régions côtières où la salinité du sol et de l'eau d'irrigation sont une réalité. Le stress salin est connu pour ses effets négatifs sur la croissance et le développement de la plante à tous les stades de développement (Ould Mohamdi et al., 2011; Gandonou et Skali Senhaji, 2015). La production des plantes dans des zones salines dépend largement du succès de la germination, de la levée, de l'établissement de la plante et de l'efficience de la phase 
reproductive (Akinci et al., 2004). Il a été rapporté que la salinité provoque une modification importante de la concentration de certaines molécules osmo-régulatrices chez les plantes (Gandonou et al., 2011) et celles des composés bioactifs des fruits de tomate (Zhang et al., 2016) conduisant ainsi à une modification de sa qualité nutritionnelle comme ceci est généralement le cas pour tous les stress environnementaux chez les légumes (Prasad et al., 2014). Ainsi, un stress salin excessif réduit la taille des fruits de tomate, la productivité et augmente le temps de floraison (Saito et al., 2006), pendant qu'un stress salin modéré améliore la qualité des fruits par l'augmentation des taux de caroténoïdes, de solides solubles totaux (sucres, acides organiques, aminoacides); ce qui relève le goût de la tomate (De Pascale et al., 2001 ; Krauss et al., 2006). Le stress salin influence également la teneur en vitamines et en lycopène, ce qui a d'implication sur la physiologie des végétaux (Ali et Ismail, 2014).

Malgré une littérature abondante sur les effets du stress salin chez la tomate, seulement quelques rares études se sont intéressées à la réponse des cultivars de tomate produits au Bénin. Dans une étude récente, nous avons démontré que la salinité a réduit la croissance des plantes chez sept cultivars de tomate produits au Bénin et qu'il existe une variabilité dans la résistance relative au stress salin de ces cultivars (Kinsou et al., 2020). Comme la tomate est principalement utilisée au Bénin comme légume-fruit, il est important de savoir dans quelle mesure la salinité agit sur la floraison, la production de fruits et si elle modifie la concentration en composés nutritionnels des fruits. La présente étude visait à évaluer l'effet de la salinité sur la floraison, le nombre de fruit, la taille des fruits, la masse fraîche des fruits de même que sur la concentration en hydrates de carbone, en lycopène, et en vitamines $\mathrm{B}$ et $\mathrm{C}$ de l'un des cultivars locaux les plus cultivés au Bénin.

\section{MATERIEL ET METHODES}

Matériel végétal

L'étude a porté sur le cultivar Akikon, cultivar local du Bénin identifié comme sensible à la salinité au stade jeune plant (Kinsou et al., 2020). Les semences ont été fournies par le Sous- Programme Cultures Maraichères de l'Institut National des Recherches Agricoles du Bénin (INRAB).

\section{Conditions expérimentales}

L'expérience a été réalisée dans une serre à l'Institut National des Recherches Agricoles du Bénin (INRAB)/ (AbomeyCalavi, République du Bénin). Les plantes ont été cultivées à une température de $26{ }^{\circ} \mathrm{C}$ jour $/ 22^{\circ} \mathrm{C}$ nuit avec la lumière naturelle et une humidité relative de $55 \%$. Les graines ont été mises en germination dans des bacs remplis de terreau pendant deux semaines. Les jeunes plants ont été ensuite transférés dans des petits pots de $5,8 \mathrm{~cm}$ de diamètre et de $6 \mathrm{~cm}$ de hauteur contenant un mélange de terreau et de sable 50: 50 (une plante/pot) et cultivés pendant une semaine avant l'application du stress. Les plants de trois semaines ont été soumis, dans des grands pots remplis de terre du même mélange à quatre concentrations de $\mathrm{NaCl}: 0 ; 30 ; 60$ et $120 \mathrm{mM}$ de $\mathrm{NaCl}$ correspondant respectivement à des conductivités électriques de 0,$221 ; 3,827$; 6,47 et $14,02 \mathrm{dS} . \mathrm{m}^{-1}$ par arrosage tous les deux jours avec $100 \mathrm{ml} /$ pot.

\section{Evaluation de la floraison}

La date d'apparition des premières fleurs a été notée pour chaque traitement. Ainsi, le nombre de jours mis par chaque plante pour sortir sa première fleur à partir du jour d'imposition du stress a été déterminé.

\section{Détermination de la maturation des fruits, du nombre, de la taille et de la masse fraîche des fruits}

La date d'apparition des premiers fruits mûrs (ayant atteint la maturité physiologique 
matérialisée par le début de changement de couleur) a été notée pour chaque traitement. Cette date a permis de déterminer le nombre de jours mis par chaque plante pour donner son premier fruit mûr à partir du jour d'imposition du stress.

Les fruits mûrs ont été collectés pour chaque traitement et comptés 88 jours après l'application du stress. Les premiers fruits mûrs provenant de chaque traitement ont été photographiés et dix fruits ont été sélectionnés de chaque plante et pesés. La masse fraîche moyenne de trois fruits de chaque traitement a été obtenue en calculant la moyenne des trois valeurs obtenues; soit à partir de neuf (9) fruits pour chaque traitement.

\section{Détermination des concentrations en composés nutritionnels}

Les concentrations des composés nutritionnels ont été déterminées sur des fruits mûrs collectés 15 jours après la maturation du premier fruit. Les concentrations de sucres totaux et réducteurs ont été déterminées par la méthode de Dubois et al. (1965), les protéines avec la méthode de Gornall (1949), les vitamines B6 et B12 par la méthode de Sami et al. (2014) avec de petites modifications; la vitamine $\mathrm{C}$ avec la méthode de Karboue et Nesrallah (2014), et le lycopène par la méthode de Benakmoum et al. (2008) modifiée.

\section{Analyse statistique}

Pour tous les paramètres, les valeurs sont présentées sous la forme moyenne \pm erreur standard. L'analyse des principaux effets de l'intensité du stress a été basée sur l'analyse de la variance à une voie (ANOVA) pour les variables quantitatives. Les moyennes ont été comparées en utilisant le test de Turkey à $5 \%$. Les analyses statistiques ont été réalisées en utilisant le logiciel JMP Pro 12 (SAS Institute, 2015). Pour les variables qualitatives (nombre de jours et de fruits), l'analyse des principaux effets de l'intensité du stress a été basée sur le test non paramétrique. Les différences entre les moyennes ont été comparées grâce au test de Kruskal-Wallis. Les analyses statistiques de ces variables ont été réalisées grâce au logiciel Minitab (Minitab, 2014).

\section{RESULTATS}

\section{Effet de $\mathrm{NaCl}$ sur la floraison et la maturation des fruits}

Le Tableau 1 présente les dates d'apparition des premières fleurs et de maturation des premiers fruits pour chaque concentration de $\mathrm{NaCl}$. L'effet de la salinité s'est traduit par un allongement de la date d'apparition des premières fleurs passant de 9 jours après l'application du stress chez le témoin à environ 11 jours avec 30, 60 et à 13 à $120 \mathrm{mM} \mathrm{NaCl}$. Mais cet effet du $\mathrm{NaCl}$ n'est pas significatif. En ce qui concerne la date de maturation des fruits, l'effet de la salinité s'est traduit également par un léger allongement non significatif de la date de maturation des premiers fruits passant d'environ 40 jours après l'application du stress chez le témoin et atteignant son maximum de 43 jours à $120 \mathrm{mM}$ $\mathrm{NaCl}$. La salinité n'a donc eu aucun effet sur les dates d'apparition des premières fleurs et de maturation des premiers fruits.

\section{Effet du NaCl sur le nombre de fruits}

Le stress salin a provoqué une réduction significative $(\mathrm{p}<0,05) \mathrm{du}$ nombre de fruits (Tableau 2). Ce nombre est passé d'environ $7,67 \mathrm{chez}$ le témoin à 5 à $30 \mathrm{mM} \mathrm{NaCl}$; à environ 5,67 à $60 \mathrm{mM}$ puis à 3,33 à $120 \mathrm{mM}$ $\mathrm{NaCl}$. La salinité a donc réduit le nombre de fruits produits chez le cultivar Akikon.

\section{Effet du NaCl sur la masse fraîche des fruits}

L'effet de la salinité s'est traduit par une réduction significative de la masse fraîche des fruits (Figure 1). Les réductions de l'ordre de $40,259 \%$; $40,667 \%$ et $52,96 \%$ par rapport au témoin respectivement pour les concentrations de $30 ; 60$ et $120 \mathrm{mM}$ de $\mathrm{NaCl}$. La réduction est significative $(\mathrm{p}<0,001)$ à partir de $30 \mathrm{mM}$. 
Effet du $\mathrm{NaCl}$ sur les teneurs en sucres totaux, sucres réducteurs et en protéines des fruits

L'effet de la salinité s'est traduit par une réduction significative $(\mathrm{p}<0,001)$ de la concentration de sucres totaux et de sucres réducteurs à partir de $30 \mathrm{mM} \mathrm{NaCl}$ (Figures 2 et 3). Les plus fortes réductions ont été observées à $60 \mathrm{mM} \mathrm{NaCl}$ respectivement $68 \%$ et $33 \%$ pour les sucres totaux et sucres réducteurs. La salinité n'a pas eu d'effet significatif sur les teneurs en protéines des fruits (Figure 4).
Effet de $\mathrm{NaCl}$ sur les concentrations de vitamines et de lycopène

La salinité a provoqué une augmentation significative $(\mathrm{p}<0,001)$ à partir de $30 \mathrm{mM} \mathrm{NaCl}$ des concentrations de vitamines B6, B12 et C (Figures 5, 6 et 7) de même que celle du lycopène (Figure 8 ) dans les fruits de tomate. L'augmentation est de l'ordre de $45 \% ; 31 \%$ et $77 \%$ respectivement pour la vitamine $\mathrm{B} 6, \mathrm{~B} 12$ et $\mathrm{C}$ à $30 \mathrm{mM}$ de $\mathrm{NaCl}$; et $38 \% ; 34 \%$ et $67 \%$ respectivement à $120 \mathrm{mM}$ $\mathrm{NaCl}$. Pour le lycopène, l'augmentation est de l'ordre de $66 \% ; 82 \%$ et $386 \%$ respectivement en présence de $30 ; 60$ et $120 \mathrm{mM}$ de $\mathrm{NaCl}$. Ainsi, l'augmentation est plus marquée pour le lycopène que les vitamines étudiées.

Tableau 1: Date d'apparition des premières fleurs et de maturation des fruits suivant les concentrations de $\mathrm{NaCl}$ chez le cultivar Akikon.

\begin{tabular}{lll}
\hline Traitements & D A P F & D M F \\
\hline $00 \mathrm{mM}$ & $9 \pm 0,577 \mathrm{a}$ & $39,667 \pm 1,667 \mathrm{a}$ \\
$30 \mathrm{mM}$ & $11 \pm 0,5774 \mathrm{a}$ & $42,667 \pm 0,667 \mathrm{a}$ \\
$60 \mathrm{mM}$ & $11 \pm 1,5275 \mathrm{a}$ & $43,667 \pm 1,33 \mathrm{a}$ \\
$120 \mathrm{mM}$ & $13,333 \pm 1,201 \mathrm{a}$ & $43 \pm 1,155 \mathrm{a}$ \\
Prob>F & 0,0986 & 0,2002
\end{tabular}

Les moyennes avec des lettres différentes à l'intérieur des colonnes sont significativement différentes $(\mathrm{p}<0,05)$.

Tableau 2 : Nombre de fruits murs par plante de tomate (Cv. Akikon) en présence de différentes concentrations de $\mathrm{NaCl}$.

\begin{tabular}{cc}
\hline Concentrations de $\mathbf{N a C l}(\mathbf{m M})$ & Nombre de fruits mûrs/plant \\
\hline 0 & $7,67 \pm 0,729 \mathrm{a}$ \\
30 & $5 \pm 1,47 \mathrm{ab}$ \\
60 & $5,67 \pm 0,839 \mathrm{ab}$ \\
120 & $3,333 \pm 0,385 \mathrm{~b}$ \\
\hline
\end{tabular}

Les moyennes avec des lettres différentes sont significativement différentes $(\mathrm{p}<0,05)$. 


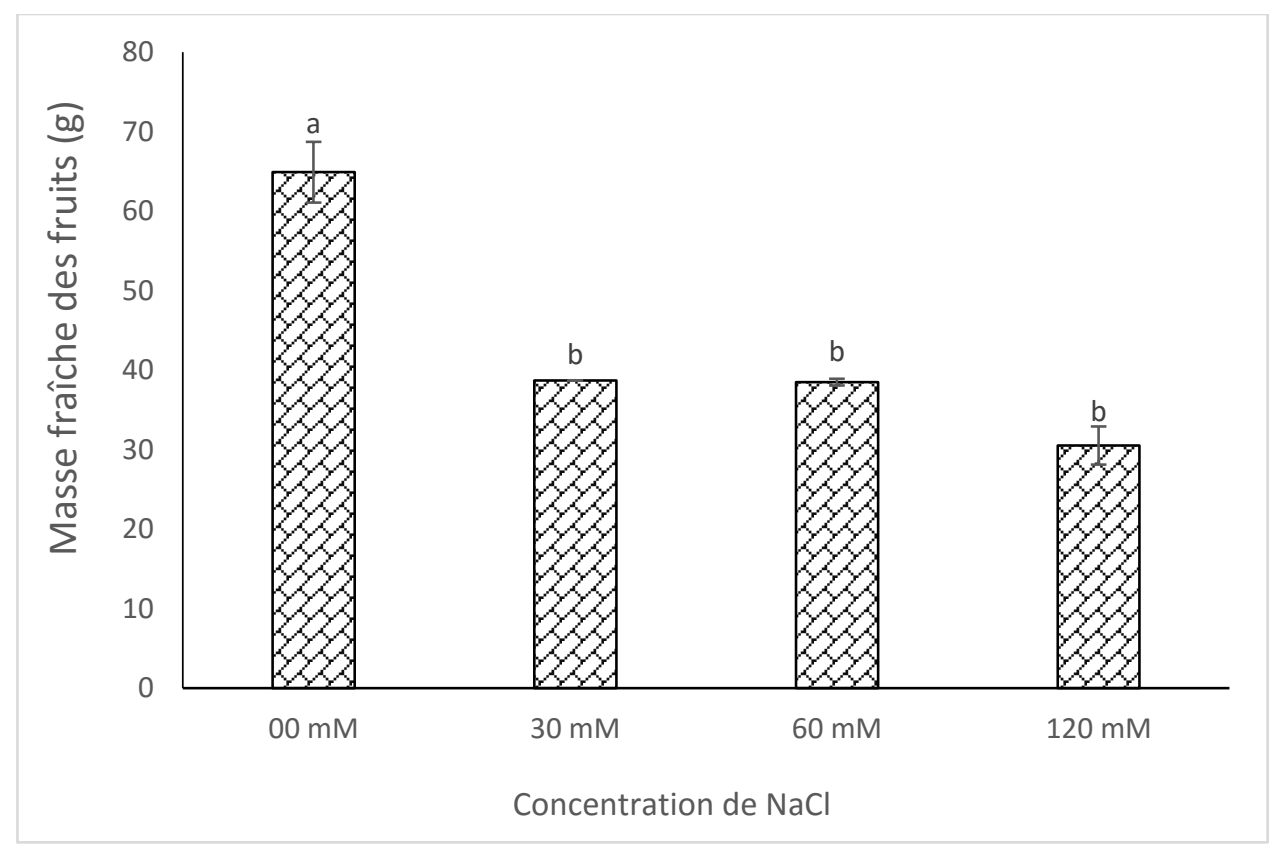

Figure 1 : Masse fraîche de trois fruits mûrs (en g) de tomate (Cv. Akikon) en présence de différentes concentrations de $\mathrm{NaCl}(\mathrm{n}=3)$. Les moyennes avec des lettres différentes sont significativement différentes $(\mathrm{p}<0,001)$.

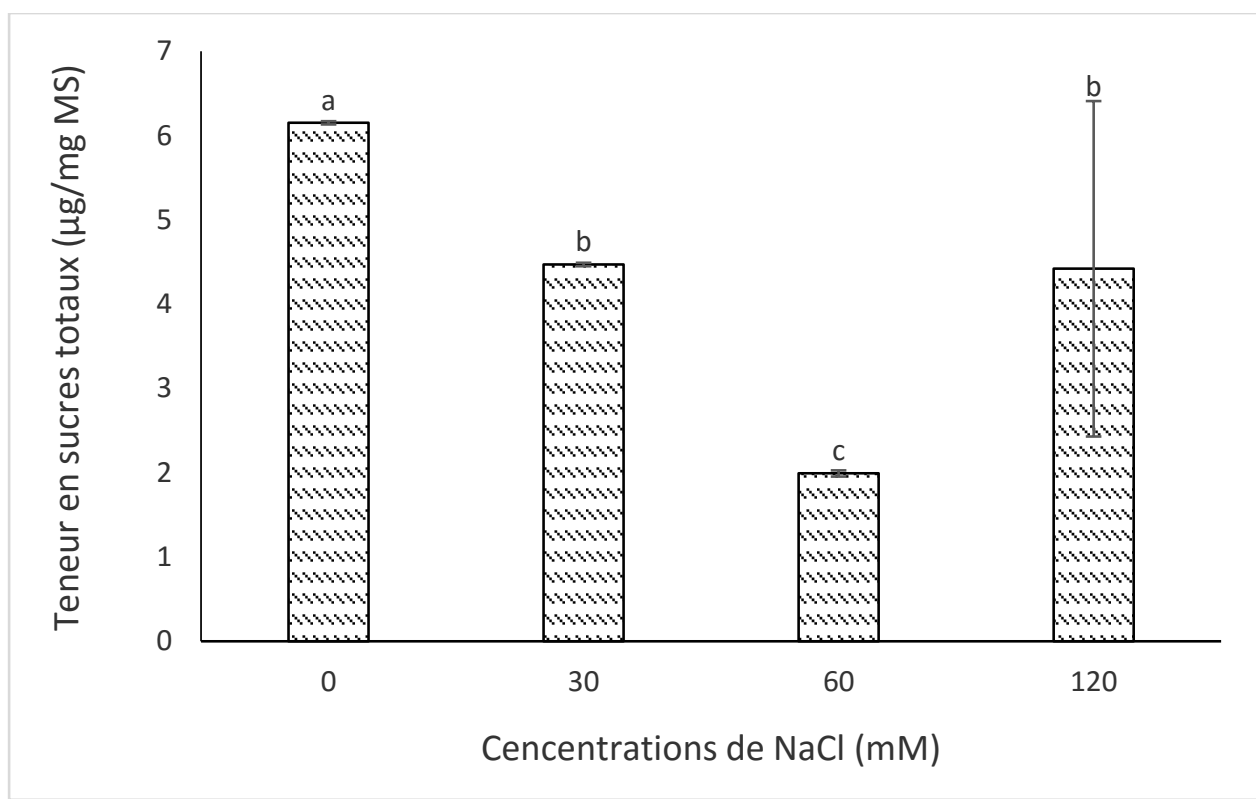

Figure 2: Concentrations en sucres totaux des fruits de tomate (Cv. Akikon) en fonction des concentrations de $\mathrm{NaCl}(\mathrm{n}=3)$. Les moyennes avec des lettres différentes sont significativement différentes $(\mathrm{p}<0,001)$. 


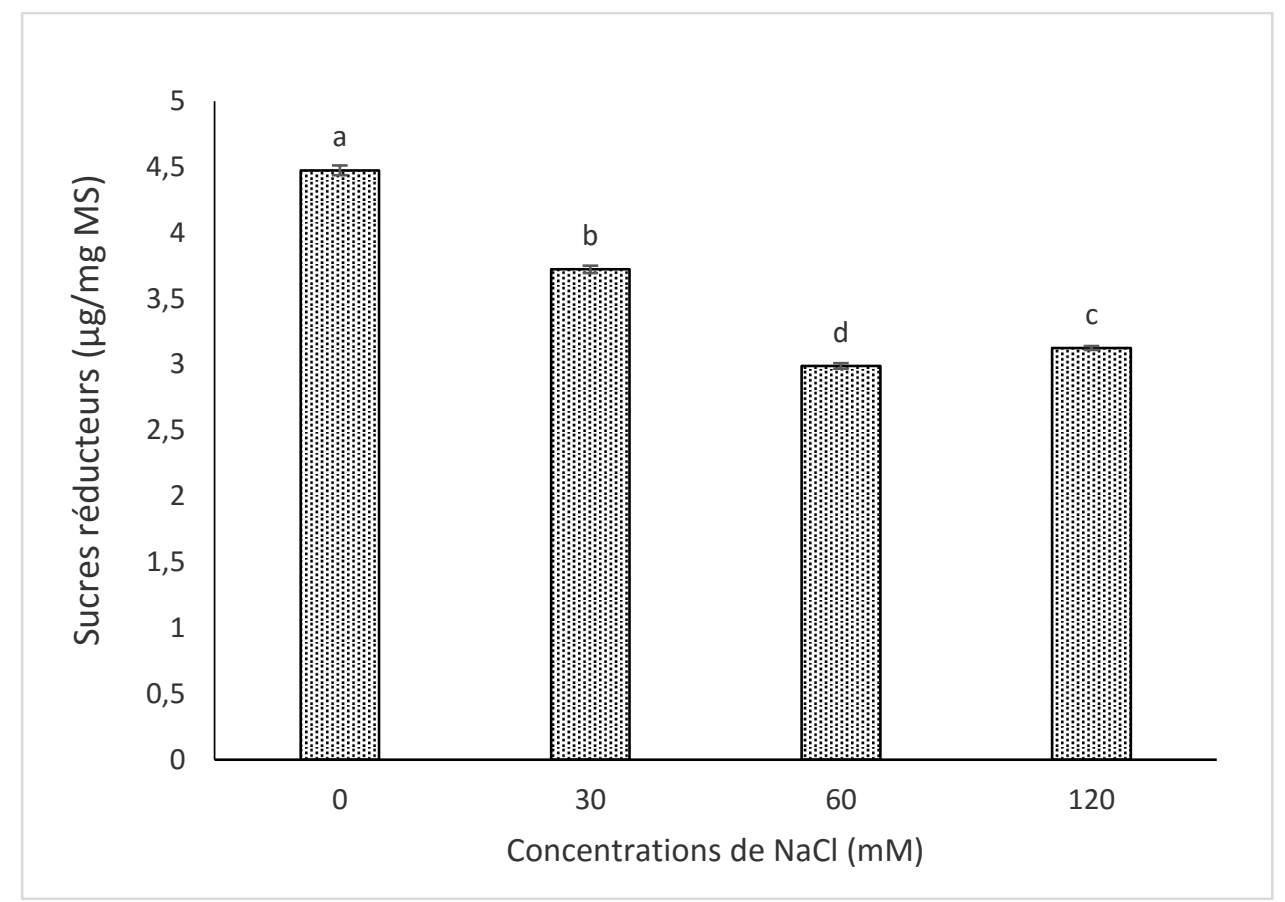

Figure 3 : Concentrations en sucres réducteurs des fruits de tomate (Cv. Akikon) en fonction des concentrations de $\mathrm{NaCl}(\mathrm{n}=3)$. Les moyennes avec des lettres différentes sont significativement différentes $(\mathrm{p}<0,001)$.

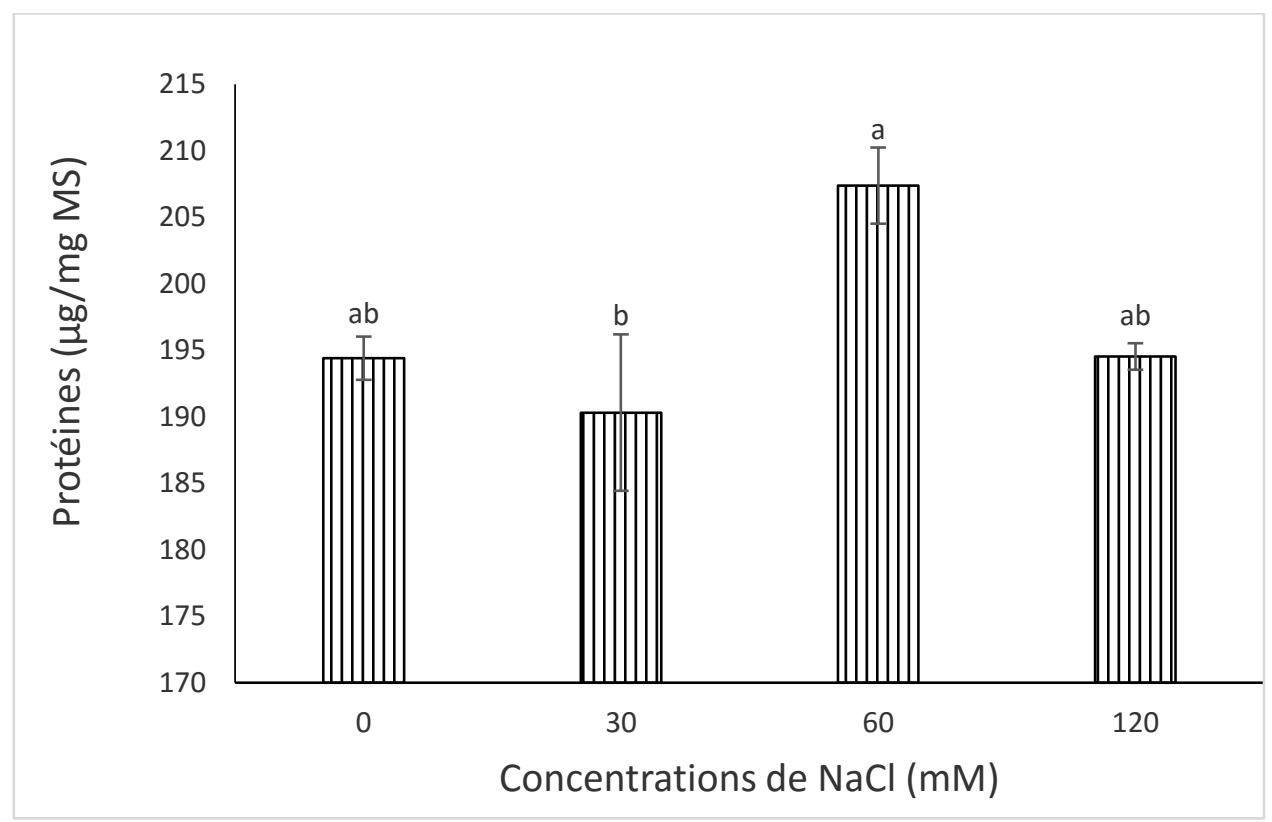

Figure 4: Concentrations en protéines des fruits de tomate (Cv. Akikon) en fonction des concentrations de $\mathrm{NaCl}(\mathrm{n}=3)$. Les moyennes avec des lettres différentes sont significativement différentes $(\mathrm{p}<0,001)$. 


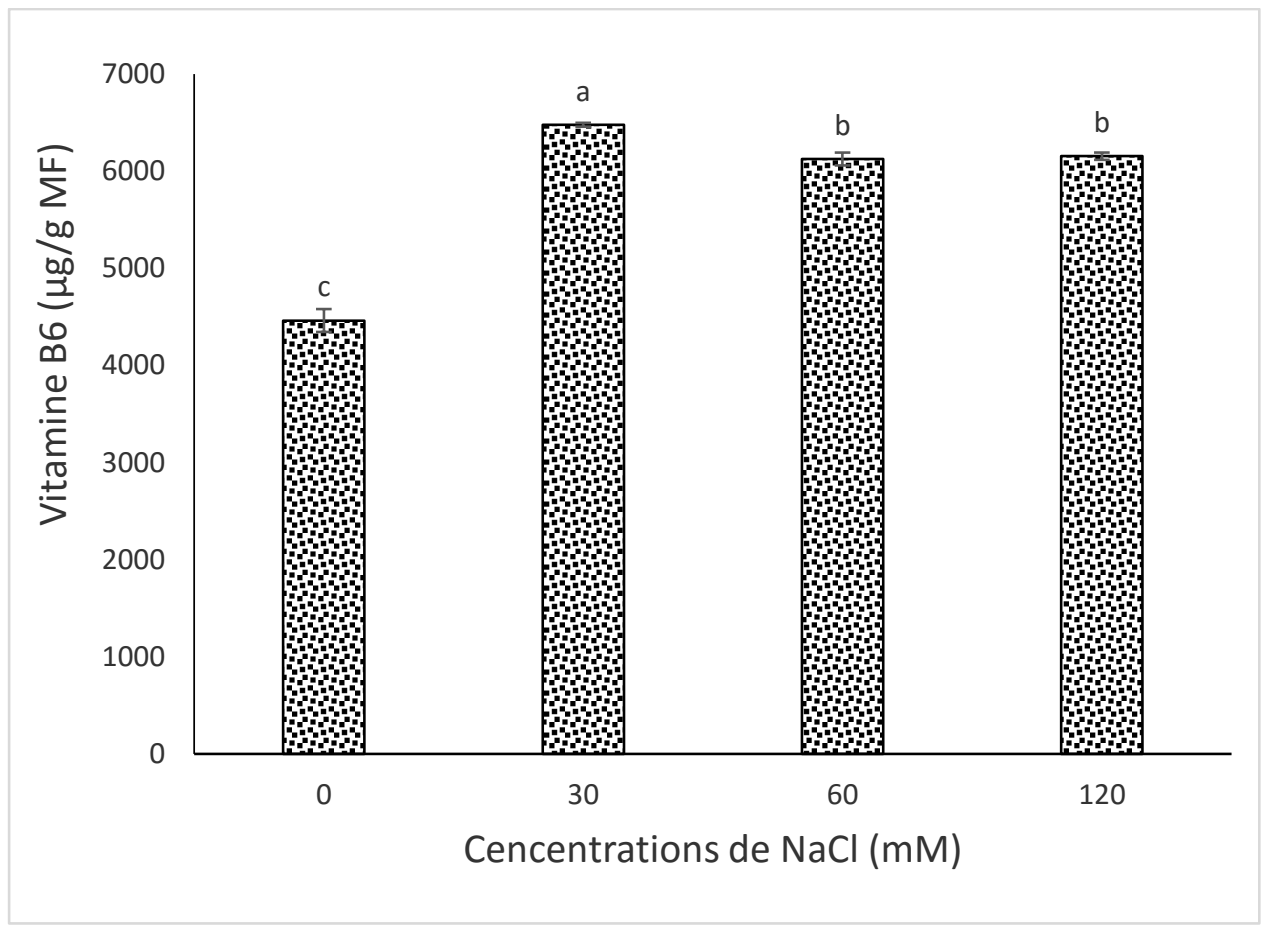

Figure 5 : Concentrations en vitamine B6 des fruits de tomate (Cv. Akikon) en fonction des concentrations de $\mathrm{NaCl}(\mathrm{n}=3)$. Les moyennes avec des lettres différentes sont significativement différentes $(\mathrm{p}<0,001)$.

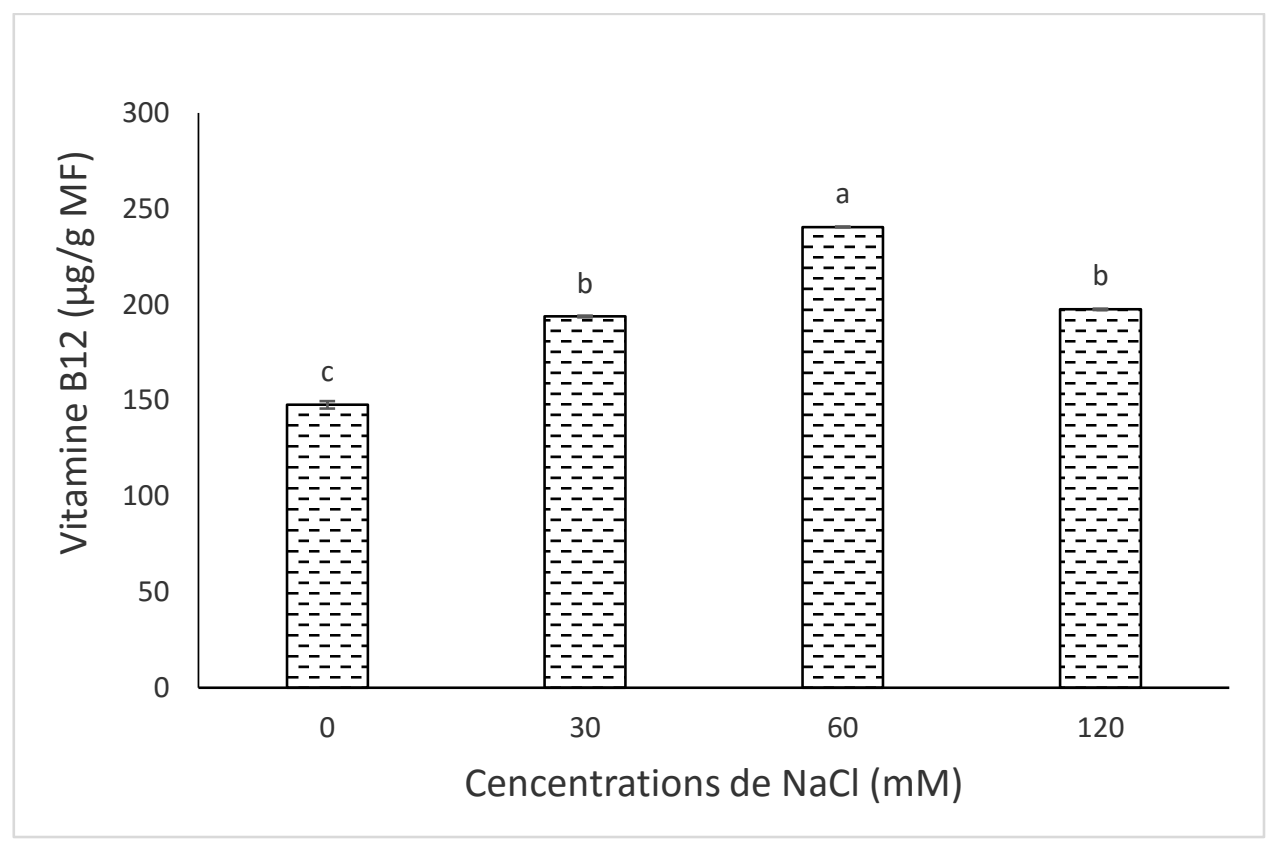

Figure 6 : Concentrations en vitamine B12 des fruits de tomate (Cv. Akikon) en fonction des concentrations de $\mathrm{NaCl}(\mathrm{n}=3)$. Les moyennes avec des lettres différentes sont significativement différentes $(\mathrm{p}<0,001)$. 


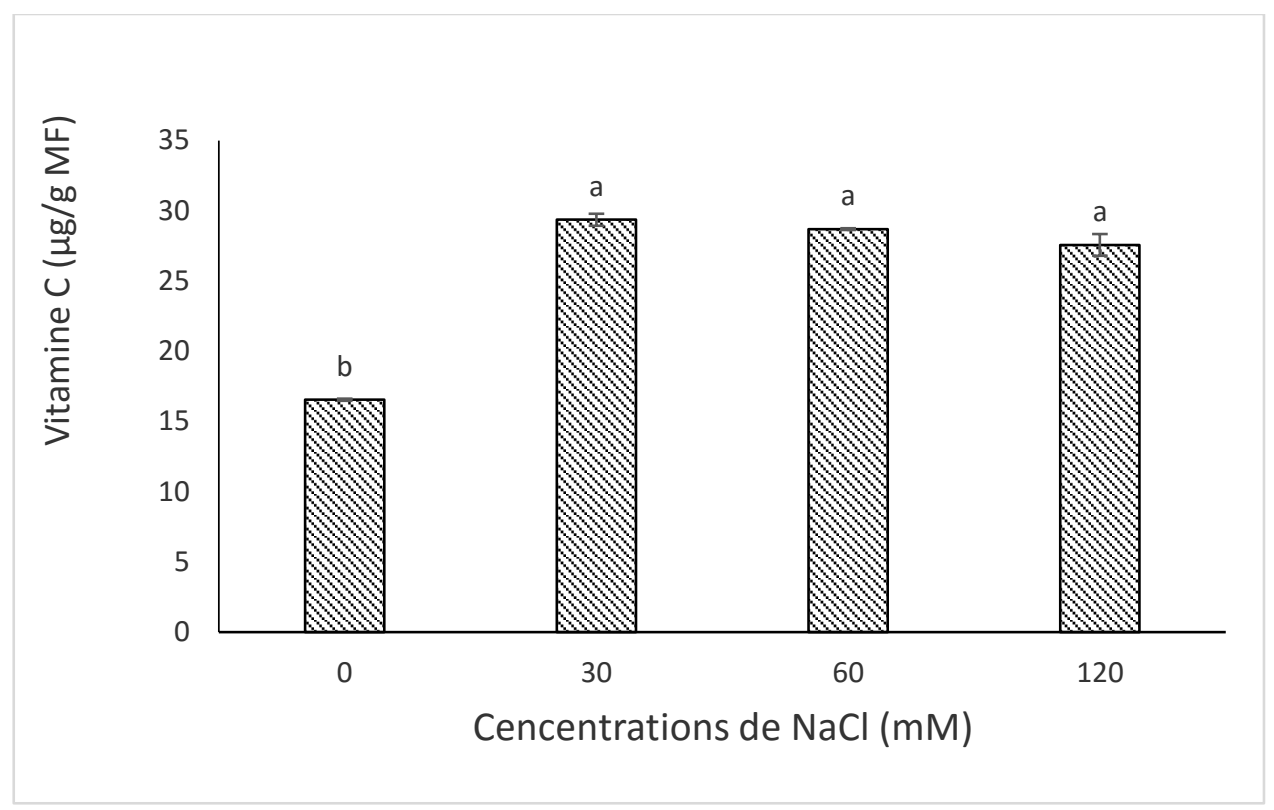

Figure 7 : Concentrations en vitamine $\mathrm{C}$ des fruits de tomate (Cv. Akikon) en fonction des concentrations de $\mathrm{NaCl}(\mathrm{n}=3)$. Les moyennes avec des lettres différentes sont significativement différentes $(\mathrm{p}<0,001)$.

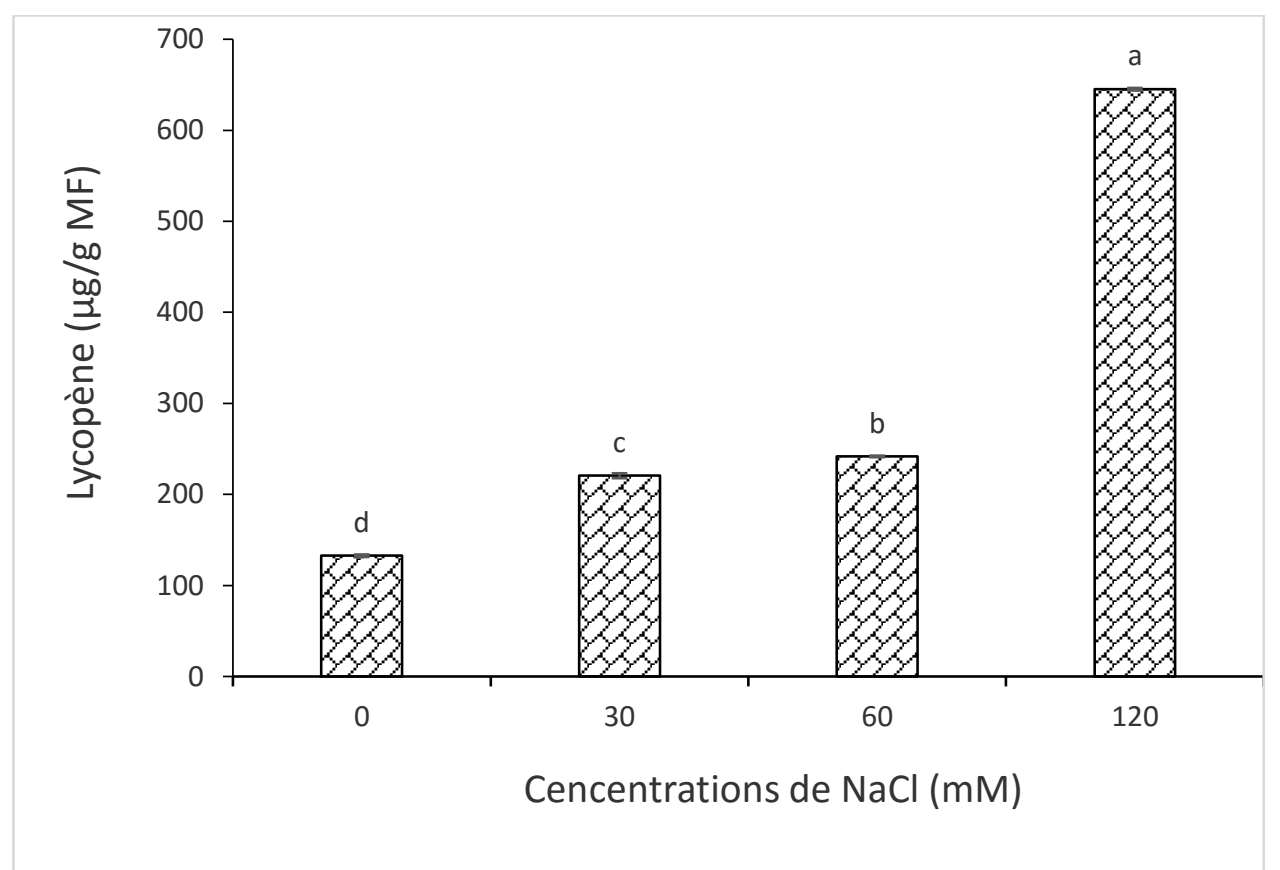

Figure 8: Concentrations en lycopène des fruits de tomate (Cv. Akikon) en fonction des concentrations de $\mathrm{NaCl}(\mathrm{n}=3)$. Les moyennes avec des lettres différentes sont significativement différentes $(\mathrm{p}<0,001)$. 


\section{DISCUSSION}

\section{Effet de la salinité sur la floraison et la fructification}

Cette étude a montré que le stress salin n'influence pas significativement la date d'apparition des premières fleurs chez le cultivar Akikon. Chez diverses espèces, Van Zandt et Mopper (2002), Zapryanova et Atanassova (2009) et Kpinkoun et al. (2019) ont prouvé qu'il y a une augmentation du temps d'apparition des premières fleurs sous l'effet de la salinité traduisant un retard de la floraison. La réponse de la floraison à ce niveau semble dépendre de l'espèce et probablement du génotype considéré à l'intérieur de chaque espèce comme c'est généralement le cas pour les autres paramètres de croissance et de développement.

L'étude a également montré que le stress salin n'influence pas significativement la date de maturation des fruits chez le cultivar Akikon. Cependant, chez le cultivar de tomate Buran F1, Pašalić et al. (2016) ont rapporté que le $\mathrm{NaCl}$ a réduit le temps de maturation des fruits. La présente étude a montré que la taille et la masse fraîche des fruits ont diminué avec l'augmentation de la salinité. Ces résultats corroborent ceux d'Assimakopoulou et al. (2015) et d'Abdelgawad et al. (2019) qui ont montré qu'il y a une réduction du poids moyen et du diamètre des fruits de tomate sous l'effet de la salinité. Des résultats similaires ont été également rapportés par Ali et Ismail (2014) ; Saito et Matsukura (2014) et Zhang et al. (2016) chez d'autres variétés de tomate. Les présents résultats ont également montré que la salinité a réduit significativement le nombre de fruits. Une observation similaire a été faite par Parvin et al. (2015) chez d'autres variétés de tomate. Chez des cultivars de piment, Kpinkoun et al. (2019) ont trouvé qu'il y a une diminution du nombre et du poids des fruits au fur et à mesure que la concentration de $\mathrm{NaCl}$ augmente. Toujours chez le piment, Navarro et al. (2010) ont remarqué qu'il y a eu une diminution du poids de fruits de piment ; alors que le nombre de fruits a augmenté. La même tendance a été rapportée chez le concombre (Trajkova et al., 2006). Les réductions de taille, de poids et de nombre vont indubitablement conduire à une baisse de rendement. Ainsi, Zhang et al. (2016) ont trouvé que la salinité a provoqué la réduction du rendement chez la tomate alors que Navarro et al. (2010) ont rapporté la même tendance chez le piment.

\section{Effet de la salinité sur la qualité nutritionnelle des fruits}

La concentration de sucres réducteurs et de sucres totaux a diminué dans les fruits du cultivar Akikon sous l'effet de la salinité. Cette observation a été remarquée aussi par Gautier et al. (2010) chez la variété Cervil où il y a eu diminution non significative de teneur en sucres totaux dans les fruits murs de tomate. Ces mêmes auteurs ont constaté que le stress salin a stimulé la synthèse des sucres solubles chez d'autres variétés de tomate telles que Levovil et Marmara. Ces résultats révèlent une différence entre la réponse de différentes variétés de tomate vis-à-vis de la salinité en ce qui concerne l'accumulation des sucres et indiquent une variabilité de la réponse en fonction des génotypes. La salinité a également provoqué l'augmentation significative de la concentration de lycopène chez le cultivar Akikon: ces résultats sont en accord avec ceux de Krauss et al. (2006) qui ont rapporté que la salinité a augmenté la teneur en lycopène des fruits de tomate. La coloration rouge de la tomate étant due à l'accumulation de caroténoïdes principalement le lycopène et le bêta-carotène (Mackinney, 2012), on peut dire que la salinité améliore la coloration rouge des fruits de tomate, et par conséquence sa qualité visuelle. Par contre, Ali et Ismail (2014) ont observé une diminution de la teneur en lycopène en fonction des concentrations de sel dans les fruits d'une autre variété de tomate 'Super Strain B'. Ce résultat indique que la réponse de la teneur en lycopène des fruits de tomate face à la salinité varie en fonction du génotype. En ce qui concerne les vitamines B6, $\mathrm{B} 12$ et $\mathrm{C}$, leur teneur a augmenté dans les fruits de tomate. Des résultats similaires ont été rapportés chez d'autres variétés de tomate par Stamatakis et al. (2003), Kim et al. (2008), Gautier et al. (2010) et Zhang et al. (2016). Cependant, Hanson et al. (2016) ont indiqué que les vitamines B qui sont des précurseurs 
métaboliques des cofacteurs sont détruites sous l'effet de la salinité. C'est ce qui a été observé par Kpinkoun et al. (2019) pour les vitamines B6 et B12 dans les fruits de piment exposé au stress salin. Par contre, chez le cultivar de tomate utilisé dans cette étude, c'est une réponse opposée qui a été observée comme cela avait déjà été observé dans les feuilles d'amarante (Wouyou et al., 2017). La réponse de la teneur en vitamines vis-à-vis de la salinité semble dépendre aussi de l'espèce que de la variété.

D'une manière générale, la salinité a induit une amélioration de la qualité nutritionnelle des fruits de tomate à travers une réduction de la teneur en sucres et une augmentation des teneurs en vitamines et en lycopène. Qaryouti et al. (2007) ont observé que chez le cultivar Durinta F1 de tomate, la qualité du fruit est améliorée avec l'augmentation du stress salin du milieu de culture. La même tendance a été rapportée chez le cultivar Pepe de tomate (Zhang et al., 2016).

\section{Conclusion}

La présente étude nous a permis de dire que la salinité n'a pas influencé la date de floraison et de maturation des fruits chez le cultivar Akikon. Elle a cependant réduit le nombre de fruits et la masse fraîche des fruits induisant une diminution de production. Le sel a également induit une réduction des teneurs en sucres dans le fruit et une augmentation de la teneur en lycopène et en vitamines B6, B12 et C. La couleur rouge des fruits est devenue plus intense du fait de la forte accumulation de lycopène. La salinité a donc provoqué une amélioration de la qualité nutritionnelle des fruits de tomate du cultivar Akikon.

\section{CONFLIT D'INTERETS}

Les auteurs déclarent qu'il n'y a aucun conflit d'intérêt.

\section{CONTRIBUTIONS DES AUTEURS}

EK a planifié l'étude, écrit le protocole et écrit la première mouture du manuscrit. EK, AMA et CBG ont géré la recherche documentaire et la revue de littérature. EK, AMA, ACGM et JKK ont contribué à la rédaction du protocole et ont géré les dosages effectués dans l'étude. EK a réalisé les analyses statistiques. FAK, HA et LL ont contribué à la rédaction du protocole. Tous les auteurs ont lu et approuvé la version finale du manuscrit.

\section{REMERCIEMENTS}

Les auteurs remercient M. Christian DANSOU du Laboratoire d'Enzymologie et de Biochimie des Protéines pour son assistance technique pour les dosages effectués.

\section{REFERENCES}

Abdelgawad KF, El-Mogy MM, Mohamed MIA, Garchery C, Stevens RG. 2019. Increasing Ascorbic Acid Content and Salinity Tolerance of Cherry Tomato Plants by Suppressed Expression of the Ascorbate Oxidase Gene. Agronomy, 9(51): 1-14. DOI: http://doi.org/10.3390/9020051

Akinci S, Yilmaz K, Akinci IE. 2004. Response of tomato (Lycopersicon esculentum Mill.) to salinity in the early growth stages for agricultural cultivation in saline environments. J. Environ. Biol., 25(3): 351-357. DOI:

Ali HEM, Ismail GSM. 2014. Tomato fruit quality as influenced by salinity and nitric oxide. Turk J. Bot., 38: 122-129. DOI: http://doi.org/10.3906/bot-1210-44

Assimakopoulou A, Nifakos K, Salmas I, Kalogeropoulos P. 2015. Growth, ion uptake, and yield responses of three indigenous small-sized greek tomato (Lycopersicon esculentum L.) cultivars and four hybrids of cherry tomato under $\mathrm{NaCl}$ salinity stress. Commun. Soil Sci. Plant Anal., 46: 2357-2377. DOI: http://doi.org/10.1080/00103624.2015.10 81924

Bhowmik D, Kumar KS, Paswan S, Srivastava S. 2012. Tomato-a natural medicine and its health benefits. Journal of Pharmacognosy and Phytochemistry, 1: 33-43.

Dembinska-Kiec A, Mikkänen O, Kiec-Wilk B, Mykkänen H. 2008. Antioxidant phytochemicals against type 2 diabetes. 
British Journal of Nutrition, 99(1): 109117. DOI: http://doi.org/10.1017/S0007114508965 79

Douakha F, Guernine F. 2013. Contribution à l'étude de la tolérance à la salinité chez quelques variétés de la tomate (Solanum lycopersicum L.). Mémoire de Master, Université 8Mai1945 Guelma, Guelma $53 \mathrm{p}$.

Gandonou CB, Skali Senhaji N. 2015. Sugarcane (Saccharum sp.) salt tolerance at various developmental levels. In Abiotic Stresses in Crop Plants, Chakraborty U, Chakraborty BN (eds). CABI Publishing: United Kingdom; 102111.

Gandonou CB, Bada F, Abrini J, Skali SN. 2011. Free proline, soluble sugars and soluble proteins concentrations as affected by salt stress in two sugarcane (Saccharum sp.) cultivars differing in their salt tolerance. International Journal of Biological and Chemical Sciences, 5(6): $1488-1493 . \quad$ DOI: 10.4314/ijbcs.v5i6.23

Gautier H, Lopez-Lauri F, Massot C, Murshed R, Marty I, Grasselly D, Keller C. 2010. Impact of Ripening and Salinity on Tomato Fruit Ascorbate Content and Enzymatic Activities Related to Ascorbate Recycling. Functional Plant Science and Biotechnology, 4(S1): 66-75.

Hanson AD, Beaudoin GA, McCarty DR, Gregory JF. 2016. Does Abiotic Stress Cause Functional B Vitamin Deficiency in Plants? Plant Physiol., 172: 20822097.

Kinsou E, Mensah A, Montcho HKD, Zanklan AS, Wouyou A, Kpinkoun KJ, Assogba Komlan F, Gandonou CB. 2020. Response of seven tomato (Lycopersicon esculentum Mill.) cultivars produced in Benin to salinity stress at young plant stage. Int. J. Curr. Res. Biosci. Plant Biol., 7(8): 1-11. DOI: https://doi.org/10.20546/ijcrbp.2020.708. 001

Kim HJ, Fonseca JM, Choi J, Kubota C, Kwon DY. 2008. Salt in irrigation water affects the nutritional and visual properties of romaine lettuce (Lactuca sativa L.). J. Agric. Food Chem., 56: 3772-3776. DOI: https:// doi.org/10.1021/jf0733719

Kpinkoun KJ, Amoussa AM, Mensah ACG, Assogba-Komlan F, Lagnika L, Gandonou CB. 2019. Effect of salt stress on flowering, fructification and fruit nutrients concentration in local cultivar of chili pepper (Capsicum frutescens L.). Int. J. Plant Physiol. Biochem., 11(1): 17.

https://doi.org/10.4314/jab.v133i1.8

Krauss S, Schnitzler W, Grassmann J, Woltike M. 2006. The influence of differeent electrical conductivity values in a simplified recirculating soiless system on inner and outer fruit quality characteristics of tomato. Agric. Food Chem., 54: 441-448. DOI: https://doi.org/10.1021/jf051930a

Navarro JM, Garrido C, flores P, Martinez V. 2010. The effect of salinity on yield and fruit quality of pepper grown in perlite. Spanish Journal of Agricultural Research, 8: 142-150.

Abir M, Hannachi C, Zid E. 2006. Régénération in vitro de plantes de tomate (Lycopersicon esculentum Mill.) adaptées au NaCl. Tropicultura, 24(4): 221-228.

Ould Mohamdi M, Bouya D, Ould Mohamed Salem A. 2011. Etude de l'effet du stress salin $(\mathrm{NaCl})$ chez deux variétés de tomate (Campbell 33 et Mongal). International Journal of Biological and Chemical Sciences, $\quad$ 5(3): $\quad 860-900 . \quad$ DOI: 10.4314/ijbcs.v5i3.72171

Parvin K, Ahamed KU, Islam MM, NazmulHaque MD. 2015. Response of tomato plant under salt stress: role of exogenous calcium. Journal of Plant Sciences, 10: 222-233.

DOI: https://doi.org/10.3923/jps.2015.222.233

Pascale SD, Maggio A, Fogliano V, Ambrosino P, Ritieni A. 2001. Irrigation with saline water improves carotenoids content and antioxidant activity of tomato. J. Hortic. Sci. Biotechnol., 76: 447-453. DOI: https://doi.org /10.1080/14620316.2001.11511392 
Pašalić $\mathrm{B}$, Todorović $\mathrm{V}$, KolešKa I, Basancic B, Đekić N. 2016. Effect of salinity on color changes, sugar and acid concentration in tomato fruit. Agriculturae Conspectus Scientificus, 81(3): 137-142.

Prasad SM, Parihar P, Singh VP. 2014. Effect of Salt Stress on Nutritional Value of Vegetables. Biochem Pharmacol., 3: e160. DOI: https://org.doi/10.4172/2167$0501.1000 \mathrm{e} 160$

Qaryouti MM, Hamdan H, Edwan M, Hurani OM. 2007. Evaluation and characterization of Jordan Tomato Landraces. Dirasat, Agri. Scien., 34(1-2): 44-56.

Saito T, Fuduka N, Nishimura S. 2006. Effects of salinity treatment duration and planting density on size and sugar content of hydroponically grown tomato fruits. $J$. Jpn. Soc. Hort. Sci., 75: 392-398. DOI: https://doi.org/10.2503/jjshs.75.392

Saito T, Matsukura C. 2014. Effect of Salt Stress on the Growth and Fruit Quality of Tomato Plants. In Abiotic Stress Biology in Horticultural Plants, Kanayama Y, Kochetov A (eds). Springer: Japan; 3-16. DOI: https:// doi.org/10.1007/978-4-43155251-2_1

Stamatakis A, Papadantonakis N, Savvas D. 2003. The effects of silicon added to the nutrient solution either at a standard electrical conductivity. Acta Hortic., 609: 141-147.

DOI: https://doi.org/10.17660/2003.609.18
Trakjova F, Papadantonakis N. 2006. Comparative effects of $\mathrm{NaCl}$ and $\mathrm{CaCl}_{2}$ on cucumber grown in closed hydroponic system. HortScience, 41(2): 437-441.

Van Zandt PA, Mopper S. 2002. Delayed and Carryover Effects of Salinity on Flowering in Iris hexagona (Iridaceae). American Journal of Botany, 89(11): 1847-1851.

DOI: https://doi.org/10.4124111

Wouyou AD, Ahissou EA, Gandonou CB, Assogba-Komlan F, Houngbèmè A, Gbaguidi FA, Ahissou H, Lagnika L, Zanklan SA, Lutts S. 2017. Salinity increased vitamins concentration in Amaranthus cruentus leaves. Afr. J. Biotechnol., 16(44): 2106-2111. DOI: https://doi.org/10.5897/2017.16203

Zapryanova N, Atanassova B. 2009. Effects of Salt Stress on Growth and Flowering of Ornamental Annual Species. Biotechnology \& Biotechnological Equipment, 23(1): 177-179. DOI: https://doi.org/10.1080/13102818.2009.1 0818394

Zhang P, Senge M, Dai Y. 2016. Effects of salinity stress on growth, yield, fruit quality and water use efficiency of tomato under hydroponics system. Reviews in Agricultural Science, 4: 46- 55. DOI: https://doi.org/10.7831/ras.4.46 